\title{
Perspectivas del enfoque socioecológico en la conservación, el aprovechamiento y pago de servicios ambientales \\ de los bosques templados de México
}

\section{Perspectives of the socioecological approach in the preservation, utilization and the payment of environmental services of the temperate forests of Mexico}

\author{
Leopoldo Galicia”, Bruno Manuel Chávez-Vergara2,5, Melanie Kolb', Rosa Isela Jasso-Flores', Laura A. Rodríguez- \\ Bustos', Lesly Elizabeth Solís', Vidal Guerra de la Cruz³, Enrique Pérez-Campuzano' y Antonio Villanueva ${ }^{4}$
}

\begin{abstract}
Universidad Nacional Autónoma de México. Instituto de Geografía. Departamento de Geografía Física. Ciudad de México, México.

2 Universidad Nacional Autónoma de México. Instituto de Geología. Ciudad de México, México.
\end{abstract}

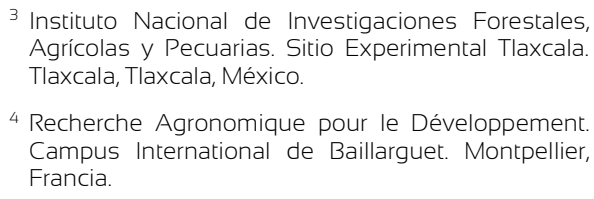

Instituto Nacional de Investigaciones Forestales Agrícolas y Pecuarias. Sitio Experimental Tlaxcala. Tlaxcala, Tlaxcala, México.

${ }^{4}$ Recherche Agronomique pour le Développement. Campus International de Baillarguet. Montpellier, Francia.

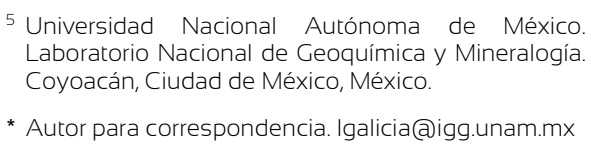

* Autor para correspondencia. Igalicia@igg.unam.mx

\section{RESUMEN}

Los bosques templados de México representan la distribución más sur de este ecosistema en el hemisferio norte y constituyen alrededor de $20 \%$ de la cobertura forestal de México, lo que los hace la clase de bosques más ampliamente distribuida. No obstante la importancia de su extensión geográfica es el tipo de vegetación menos estudiado desde el punto de vista socioecológico. El enfoque de estudio que ha predominado en este tipo de vegetación es el silvícola, guiado por la visión de mantener un flujo continuo de madera para el mercado e ingresos de los propietarios forestales, sin considerar la provisión de otros beneficios y servicios ecosistémicos. En este estudio se propone la integración de un enfoque socioecológico que promueva integrar las dependencias sociedad-naturaleza que ocurren en los bosques templados. En este contexto es imperativo: 1) entender la influencia de las especies de pinos y encinos de los bosques templados en la estructura y funcionamiento de estos bosques, en particular sobre la productividad primaria; 2) entender el impacto que tienen el cambio de uso del suelo, la extracción regulada y no regulada de biomasa sobre la estructura y el funcionamiento del ecosistema y 3) entender los aspectos sociales y económicos relacionados con diferentes herramientas de conservación (áreas naturales protegidas y pago de servicios ambientales), que representan esquemas de interacción sociedad-naturaleza. Se discute cómo la investigación y evaluación bajo este enfoque puede contribuir a reducir el enorme déficit en la producción maderable que persiste en México, proteger la diversidad biológica y mantener la provisión de los servicios ecosistémicos para el bienestar humano.

PALABRAS ClAVE: bosques templados, conservación, ecología de ecosistemas, manejo forestal, servicios ecosistémicos, socio-ecosistema.

\section{ABSTRACT}

The temperate forests of Mexico represent the southernmost distribution of this ecosystem in the Northern hemisphere and constitute about $20 \%$ of Mexico's forest cover, making them the most widely distributed type of forest in the country. In spite of the importance of its geographical extension, studies of this type of vegetation with a socio-ecological approach are rare. The silvicultural scientific approach has been the predominating type of studies in this ecosystem, guided by the vision of maintaining a continuous flow of timber for the market and paying regard to the income of forest owners, without considering the provision of ecosystem services and other benefits. This study recommends the integration of a socioecological approach that promotes the integration of the society-nature dependencies that occur in temperate forests. In this context, it is imperative to understand: 1) the influence of temperate pine and oak species on the structure and functioning of these forests, particularly on primary productivity; 2) the impact 
of soil, regulated and unregulated biomass harvest on the structure and functioning of the ecosystem and 3) the social and economic aspects related to different conservation tools (natural protected areas and payment for environmental services), which represent schemes of society interaction with nature. It is discussed how research and evaluation under this approach can contribute to reducing the enormous deficit in timber production that persists in Mexico, protecting biological diversity and maintaining the provision of ecosystem services for human well-being.

KEYWORDS: temperate forests, conservation, ecosystem ecology, forest management, ecosystem services, socio-ecosystem.

\section{INTRODUCCIÓN}

A escala global los bosques templados (BT) ocupan $1 \times 10^{7} \mathrm{~km}^{2}$, lo cual representa $25 \%$ del área forestal mundial y $8 \%$ de la superficie continental libre de hielo. En México, los bosques templados constituyen $20 \%$ de la cobertura forestal que los hace la clase de bosques más ampliamente distribuida. De esta proporción, 5\% es ocupado por bosques de encinos (BE), 14\% por bosques de pino (BP) y pino-encino (BPE) y 1\% por otras coníferas (Rzedowski, 1991). Los BPE están caracterizados por contener una elevada riqueza debido a que son el centro de diversificación de los géneros Pinus y Quercus a escala mundial (Nixon, 1993). Históricamente, los BP y BE poseen una tasa de deforestación anual promedio mayor a 0.5\% (Masera, Ordoñez y Dirzo, 1997a; Galicia et al., 2015), y en algunas regiones tienen mayor tasa de deforestación y de cambio de uso de suelo que las selvas (Kolb y Galicia, 2012); esto ha repercutido en la disminución de su extensión y del número de especies que albergan (Challenger, 1998; Gómez-Pompa, 1985; Rzedowski, 1978). Debido a lo anterior, los bosques templados contienen un alto número de especies amenazadas, en peligro de extinción y sujetas a protección especial por la NOM-059-SEMARNAT2010 (Diario Oficial de la Federación [DOF], 2010). Por ejemplo, los bosques de coníferas contienen un total de 453 especies en diferentes categorías de protección.

En México, los bosques templados tienen una elevada importancia al menos por cuatro consideraciones básicas, y a partir de estos cuatro ejes temáticos se ha desarrollado la investigación biológica y ecológica en estos ecosistemas (Fig. 1).

1) Importancia biológica. Albergan 50 especies de pinos y 140 especies de encinos, que en ambos casos representan $\sim 50 \%$ y $\sim 30 \%$ del total a escala mundial para cada género, respectivamente (Comisión Nacional para el Conocimiento y Uso de la Biodiversidad [Conabio], 2001; Valencia, 2004). Los principales estudios bajo esta perspectiva usan el enfoque evolutivo de genética de poblaciones (Delgado et al., 1999; Aguirre-Planter, Furnier, \& Eguiarte, 2000) y diferentes aspectos botánicos (Perry, 1991; Farjon y Styles, 1997).

2) Social. En ellos se concentra la mayor parte de la población rural del país, quienes poseen cerca de 70\% de las tierras forestales de México en propiedades colectivas llamadas ejidos o comunidades. Por esta razón, los estudios sociales en este ecosistema se han concentrado en estudiar temas de gobernanza (Ruppert y Antinori, 2008), organización comunitaria (Bray, Merino y Barry, 2007) y silvicultura basada en la cooperación social (Antinori y Rausser, 2007; Taylor, Cronkleton y Barry, 2011).

3) Económica. La mayor parte de la producción maderable nacional proviene de los bosques templados y representa $1 \%$ del Producto Interno Bruto (PIB) nacional. En este sentido, el manejo forestal comunitario ha sido una herramienta con la cual gobiernos tanto nacionales como locales, en el marco de una estrategia global, han incentivado que sean los mismos usuarios que utilicen sus prácticas para un adecuado aprovechamiento para mejorar los sistemas ecológicos y el bienestar de las comunidades (Bowler et al., 2012; Ellis et al., 2015).

4) Ambiental. Proveen diversos servicios ecosistémicos esenciales para el mantenimiento del capital natural y bienestar social en las diversas regiones forestales del país; los estudios se han concentrado en monitorear el cambio de uso del suelo, las tasas de deforestación y el patrimonio cultural del paisaje (PalacioPrieto et al., 2000; Galicia et al., 2015; Wallace et al., 2015). 


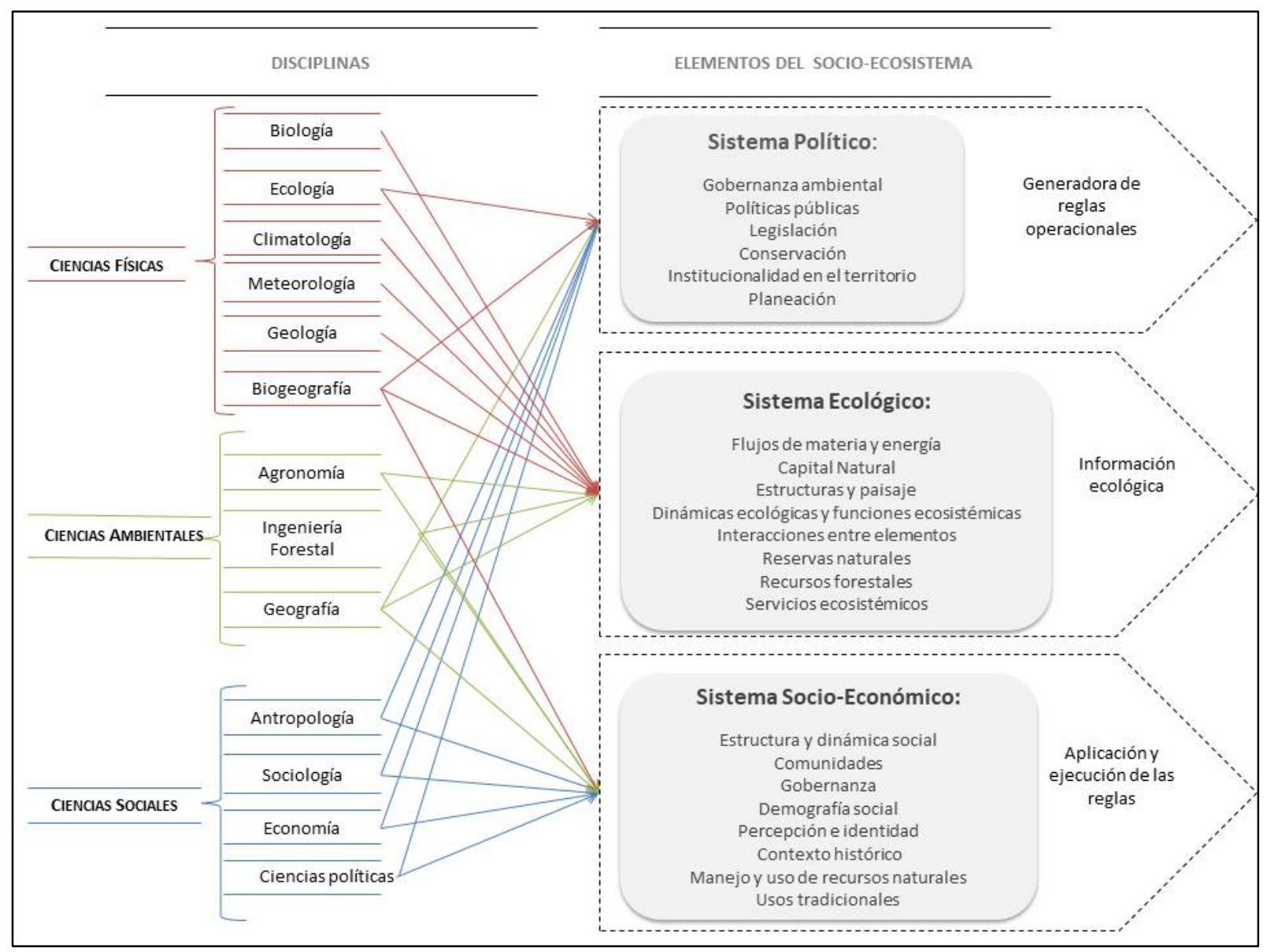

FIGURA 1. Lista de disciplinas y enfoques de investigación en los bosques templados de México.

La interacción socio-ecológica de los ecosistemas de los bosques templados a través del manejo y uso del bosque, además de políticas públicas de conservación como las áreas naturales protegidas (ANP) y el pago por servicios ambientes (PSA) (Wallace et al., 2015) ha generado un sistema complejo de estudio. No obstante, los trabajos académicos que aborden nuevos marcos de análisis e innovadores en los ecosistemas templados de México son escasos. El objetivo del presente trabajo es presentar una revisión e integración de los estudios contemporáneos de ecología, manejo y conservación en los bosques templados de México que permita ampliar la visión de futuras investigaciones bajo un enfoque socioecológico. La construcción de este enfoque conceptual permitirá identificar componentes importantes para el diseño de estrategias que garanticen su conservación, promuevan su manejo sostenible y mejoren las oportunidades de ingresos y condiciones de vida de las personas que viven de los bosques templados en México.

\section{Los bosques templados de México y su importancia para los procesos ecosistémicos}

No obstante la gran extensión y la biodiversidad que albergan los bosques templados mexicanos, son los ecosistemas con menos estudios sobre ecología, estructura y funcionamiento (Galicia y Zarco-Arista, 2014); no figuran en las principales revisiones teóricas de ecología, ni en los modelos de cambio climático global y regional. En estudios contemporáneos, Augusto et al. (2014) y Vesterdal et al. (2013) Realizaron revisiones a escala global sobre la influencia de especies de Pinus y Quercus en el funcionamiento de bosques templados y boreales, pero no incluyen ninguna referencia a los bosques templados mexicanos, los cuales son 
fundamentales dadas sus particularidades como son las diferencias en el clima debido a una variación altitudinal muy amplia, la composición de especies y sus efectos en el funcionamiento de estos ecosistemas (Galicia y Zarco-Arista, 2014).

La productividad primaria del bosque es de suma importancia debido a que es una de las variables base del sistema ecológico, ya que determina todas las interacciones de la cadena trófica, así como los beneficios económicos para las comunidades rurales. Los cambios en la biomasa y sus componentes son un indicador importante de la productividad, el potencial energético y la capacidad de absorción de $\mathrm{C}$ atmosférico de los bosques (Organización de las Naciones Unidas para la Alimentación y la Agricultura [FAO], 2015). De acuerdo con cifras de la FAO (2015), durante los últimos 25 años las existencias de carbono de la biomasa forestal a escala mundial se han reducido en casi 17.4 Gt, lo que equivale a una disminución de 697 millones de toneladas anuales o aproximadamente $2.5 \mathrm{Gt}$ de dióxido de carbono liberado a la atmósfera. Dichas emisiones son el resultado del cambio de cobertura y cambio de uso del suelo, principalmente derivado de la conversión de tierras forestales a tierras agrícolas.
En los bosques templados mexicanos es característico que una o dos especies sean dominantes en amplias regiones geográficas, por lo que juegan un papel central en los procesos que determinan la estructura y funcionamiento de estos ecosistemas. Las mediciones directas sobre la productividad primaria neta (PPN) aérea en los diferentes tipos de bosques que se han documentado señalan que la PPN de los BP oscila entre $5.89 \mathrm{Mg} \mathrm{ha}^{-1} \mathrm{año}^{-1}$ y $10.78 \mathrm{Mg} \mathrm{ha}^{-1} \mathrm{año}^{-1}$ (Tabla 1) y que tienen una biomasa arbórea promedio de $\sim 154.41 \mathrm{Mg} \mathrm{ha}^{-1}$ (MendozaPonce y Galicia, 2010; Ordóñez et al., 2008; De Jong et al., 1999; Masera, Bellon y Segura, 1995; Masera, Bellon, y Segura, 1997b; Tabla 1), mientras que en los BE no se tienen datos publicados para el país (Delgadillo, 2011, Manzano et al., 2007). El carbono (C) almacenado en la biomasa arbórea en BP es de $\sim 255.46 \mathrm{Mg}$ $\mathrm{C} \mathrm{ha}^{-1} \mathrm{y}$ en el caso de los BE de $\sim 110.55 \mathrm{Mg} \mathrm{C} \mathrm{ha}^{-1}$ (De Jong et al., 1999; Masera et al., 1995; 1997b; Tabla 1). De manera general, en estos estudios solo se cuantifica el incremento de los componentes aéreos debido a las complicaciones prácticas en la evaluación de la productividad radicular. Esto puede generar un sesgo importante en la cuantificación de la biomasa forestal, ya que se ha documentado que la productividad radicular puede comprender entre $25 \%$ y $40 \%$ del total de la PPN y contener $40 \%$ del C en los bosques (Finér et al., 2011).

TABLA 1. Producción primaria neta (PPN), biomasa, producción de hojarasca, carbono en la hojarasca y carbono en el suelo en bosques templados de México.

\begin{tabular}{|c|c|c|c|c|c|c|}
\hline $\begin{array}{l}\text { PPNA (Mg } \\
\left.h a^{-1} a \tilde{n} o^{-1}\right)\end{array}$ & $\begin{array}{c}\text { Biomasa } \\
\text { arbórea } \\
\left(\mathrm{Mg} \mathrm{ha}^{-1}\right)\end{array}$ & $\begin{array}{c}\text { Cbiomasa } \\
\text { arbórea } \\
\left(\mathrm{Mg} \mathrm{ha}^{-1}\right)\end{array}$ & $\begin{array}{c}\text { Hojarasca } \\
\left(\text { Mg ha- }^{-1}\right. \\
\text { año-1) }\end{array}$ & $\begin{array}{l}\text { Chojarasa } \\
\left(M_{g} C h a^{-1}\right)\end{array}$ & $\begin{array}{c}\text { C suelo } \\
\left(\mathrm{Mg} C \mathrm{ha}^{-1}\right)\end{array}$ & Fuente \\
\hline 8.55 & 154.41 & 155.47 & 2.98 & 3.55 & 106.56 & $\begin{array}{l}\text { Acosta-Mireles et al. (2009); Cruz-Flores y } \\
\text { Etchevers-Barra (2O11); de Jong et al. (1999); } \\
\text { Delgadillo (2O11); } \\
\text { Grier et al. (1981); López-López et al (2014); } \\
\text { Navar-Cháidez y Jurado-Ybarra, (2009); } \\
\text { Ordoñez et al. (2OO8); Pérez-Suárez et al. } \\
\text { (2009); Pineda-López et al. (2O13); Reyes- } \\
\text { Carrera et al. (2012); Rocha-Loredo \& } \\
\text { Ramírez-Marcial (2012); Silva-Arredondo y } \\
\text { Návar-Cháidez (2010); Vela y Rodríguez } \\
\text { (2012). }\end{array}$ \\
\hline$S / D$ & S/D & 106.93 & 6.23 & 3.8 & 228.77 & $\begin{array}{l}\text { Cantú-Silva et al. (2013); García-Oliva et al. } \\
\text { (2014); Ordoñez et al. 2008; Rocha-Loredo } \\
\text { y Ramírez-Marcial (2012). }\end{array}$ \\
\hline
\end{tabular}


La producción de hojarasca, que es uno de los principales mecanismos por el cual las especies vegetales afectan la acumulación de C en el suelo (Binkley, 1997; García-Montiel y Binkley, 1998; Hooper et al., 2005), puede ser similar entre especies funcionalmente diferentes (Vesterdal, Clarke, Sigurdsson, y Gunsersen, 2013; Augusto et al., 2014). Una revisión de los datos publicados sobre los tres tipos dominantes de bosques templados en México estima que la producción de hojarasca en bosques templados de México es de $\approx 298 \mathrm{~g} \mathrm{~m}^{-2}$ año-1 para $\mathrm{BP}, \approx 623 \mathrm{~g} \mathrm{~m}^{-2}$ año $^{-1}$ para BPE y $\approx 778 \mathrm{~g} \mathrm{~m}^{-2}$ año $^{-1}$ para BE, lo cual implica que los bosques de latifoliadas presentan un mayor flujo de materia orgánica y nutrientes hacia el suelo (Tabla 2). En el caso de los encinos, Pérez-Suárez, Arredondo-Moreno, HuberSannwald y Vargas-Hernández (2009) encontraron que en un rodal monoespecífico de Quercus potosina se incorpora mayor cantidad de hojarasca al suelo con mayor concentración de $\mathrm{N}$ y $\mathrm{P}$ que en un rodal monoespecífico de Pinus cembroides. ChávezVergara et al. (2015) Determinaron que Quercus castanea produce mayor cantidad de hojarasca $\left(711 \mathrm{~g} \mathrm{~m}^{-2}\right.$ año-1) que Q. deserticola (450 $\mathrm{g} \mathrm{m}^{-2}$ año ${ }^{-1}$ ) pero, debido a que la primera reabsorbe más $\mathrm{N}$ y P que la segunda, disminuye la concentración de $\mathrm{N}$ y $\mathrm{P}$ en la hojarasca, de modo que existe el mismo flujo de masa de $\mathrm{N}$ y $\mathrm{P}$ al suelo, pero la calidad de la hojarasca es diferente entre las especies (Vesterdal et al., 2013).

TABLA 2. Producción de hojarasca, y contenidos de carbono, nitrógeno y fósforo en los bosques templados de México.

\begin{tabular}{lcccc}
\hline $\begin{array}{l}\text { Tipo de } \\
\text { bosque }\end{array}$ & Hojarasca & Carbono* $^{*}$ & Nitrógeno* & Fósforo* \\
\hline \multicolumn{4}{c}{$g \mathrm{~m}^{-2} \mathrm{a}^{-1}$} \\
\hline $\begin{array}{l}\text { Bosque de } \\
\text { aciculares }\end{array}$ & 298 & 162 & 1.76 & 0.13 \\
$\begin{array}{l}\text { Bosque } \\
\text { mixto** }\end{array}$ & 623 & 291 & 5.54 & 0.43 \\
$\begin{array}{l}\text { Bosque de } \\
\text { latifoliadas }\end{array}$ & 778 & 335 & 8.09 & 0.64 \\
\hline
\end{tabular}

En los BP existe mayor masa de mantillo $\left(2913 \mathrm{~g} \mathrm{~m}^{-2}\right)$ con menor producción de hojarasca, mientras que en BE existe menor masa de mantillo (1703 $\mathrm{g} \mathrm{m}^{-2}$ ) con mayor producción de hojarasca. Estas diferencias pueden ser resultado de las tasas de descomposición de cada tipo de mantillo. Estudios en BP y BE de México muestran una mayor tasa de descomposición (0.6-0.7) que las halladas en otras partes del mundo, 0.3-0.46 aciculadas y 0.08-0.47 latifoliadas (Cárdenas, 2008; Melillo, Aber y Muratore, 1982; Das y Ramakrisshnan, 1985; Rocha-Loredo y RamírezMarcial, 2009), debido a las diferencias climáticas y la composición química foliar de las especies arbóreas del país (Cárdenas, 2008; Rocha-Loredo y Ramírez-Marcial, 2009).

Por otro lado, las tasas de descomposición en BPE pueden variar ampliamente debido a la interacción entre tipos de mantillo, lo que puede acelerar o retardar el proceso. Sin embargo, no se han podido reconocer patrones consistentes sobre la descomposición de biomasa entre grupos de especies porque la investigación sobre este proceso es escasa (Xiaogai et al., 2013). Asimismo, Oostra, Majdi y Olsson (2006), así como Finér et al. (2007) sugieren que la biomasa y producción de raíces puede diferir entre las especies de árboles caducifolios y las coníferas; sin embargo, los estudios sobre el papel de las especies de los bosques templados sobre la biomasa de raíces en términos de calidad y cantidad son escasos (Augusto et al., 2014; Versterdal et al., 2014). Es evidente a partir de estos estudios, que las interacciones entre clima, suelos, especies y tipos de bosques hace necesario desarrollar esquemas y enfoques de manejo que incorporen estas diferencias para el mantenimiento de los procesos ecológicos que intervienen en el sostenimiento de la productividad forestal.

\section{Aprovechamiento silvícola}

Los bosques boreales y templados del mundo son los principales proveedores de recursos forestales (Fürstenau et al., 2007). Particularmente en México, la actividad forestal es de gran importancia para el país; tan solo para el 2014 el consumo nacional aparente de productos forestales fue de 19.2 millones de metros cúbicos, de los cuales $29.5 \%$ fue cubierto por la producción forestal nacional (Secretaría de Medio Ambiente y 
Recursos Naturales [Semarnat], 2014). En términos monetarios esto representó $0.3 \%$ del valor del PIB nacional en ese mismo año: 38065 millones de pesos (Semarnat, 2014). Cabe señalar que el valor de las exportaciones de madera y sus derivados fue de 398.2 millones de dólares, y las importaciones de 1524.5 millones de dólares, el déficit se atribuye principalmente a la importación de productos de papel (Semarnat, 2014). Esto podría explicarse porque el manejo silvícola en México se basa únicamente en la extracción de madera y a que las plantaciones forestales están simplificadas en especies de interés comercial, que en un $90 \%$ pertenecen al género Pinus. Esto ha generado extinción local de especies y reducción de la biodiversidad, e impactado sobre la disminución de la competitividad comercial de otras especies y los recursos forestales maderables y no maderables (Hoekstra et al., 2005; Nasi y Frost, 2009).

Actualmente, alrededor de 6\% de las áreas forestales están bajo algún programa de manejo silvícola; esto podría considerarse una extensión de bosque donde es posible reconocer las prácticas forestales. Sin embargo, la extracción ilícita de madera industrial se estima en alrededor de 13 millones de metros cúbicos al año (Masera Ceron y Ordóñez, 2001). Caballero-Deloya (2008) señala que el éxito de la extracción ilícita de madera se basa en la eficiente operación de la cadena productiva que incluye desde la corta furtiva, el transporte, la industrialización hasta la comercialización. Por otra parte, Caballero-Deloya (2008) y Merino y Martínez (2014) concuerdan en que las prácticas forestales legales y organizadas a escala comunitaria y ejidal son poco viables por la discrepancia entre la realidad de las prácticas forestales y las políticas de regulación excesiva que existen en torno al uso del bosque.

En México se estima que hay 8500 comunidades forestales, y que la economía de las mismas se caracteriza por la diversificación de actividades relacionadas con el uso del bosque o de la transformación de superficies a usos agrícolas y pecuarios (Merino y Martínez, 2014). Merino y Martínez (2014) señalan que menos de $50 \%$ de las comunidades encuestadas realizan aprovechamiento forestal, y en $25 \%$ de ellas tan solo la cuarta parte de los ejidatarios y comuneros realiza colecta de productos forestales. En contraste, en las comunidades que realizan actividades forestales el ingreso económico derivado de estas representa más de la mitad del ingreso familiar anual.
Si bien es cierto que el manejo forestal comunitario ha sido destacado en la literatura internacional como un mecanismo eficiente para proveer ingreso al mismo tiempo que genera prácticas de conservación de los sistemas forestales, los mecanismos por los cuales este tipo de prácticas se vuelven exitosas van más allá de la propia comunidad. En México, los factores que inciden en que el manejo forestal comunitarios sea exitoso incluyen un marco institucional claro, beneficios económicos y ecosistémicos de corto y mediano plazo, derechos de propiedad y uso (Adcharaporn Kim y Daughtery, 2006; Cronkleton, Bray y Medina ., 2011; Bowler et al., 2012), acceso equitativo, incluyendo los de género a los beneficios (Vázquez, 2015), la capacidad de adaptación de las comunidades a las condiciones externas (Ellis et al., 2015), así como una relación de confianza entre gobierno y comunidades (Segura-Warnholtz, 2014).

Los sistemas silvícolas modifican directamente la estructura y composición del bosque, ya que tienden a favorecer y seleccionar ciertas especies (Zerecero y Pérez, 1981), además de incrementar la mortalidad de individuos o grupos de especies (Negreros y Snook, 1984). Diversos estudios en el norte de México han encontrado cambios en la estructura y composición de especies arbóreas como consecuencia de largos periodos de manejo forestal. La simplificación de la estructura y la preferencia hacia las especies de mayor valor comercial puede aumentar la vulnerabilidad de estos ecosistemas al alterar procesos fundamentales como los ciclos biogeoquímicos o los regímenes de disturbios, induciendo una pérdida de la resiliencia a los cambios ambientales en el largo plazo (Bruelheide y Luginbühl, 2009; Boiffin y Munson, 2013; Harvey y Holzman, 2014; Seidl, Rammer y Spies, 2014). A pesar de ello, el impacto de la cosecha de biomasa sobre la reducción o eliminación de la complejidad estructural, la simplificación de hábitats y los mecanismos de facilitación no han sido explorados en estudios dentro de los bosques templados mexicanos.

Las investigaciones del impacto de la cosecha de biomasa sobre las respuestas de la vegetación asociada (composición y riqueza de las especies de plantas del sotobosque y el estrato herbáceo) y algunas condiciones microambientales han sido consideradas como el único factor de alteración de la estructura y funcionamiento de estos ecosistemas (Bruelheide y Luginbühl, 
2009; Boiffin y Munson, 2013; Harvey y Holzman, 2014; Seidl et al., 2014), sin considerar sus consecuencias en otros componentes del ecosistema como suelo, clima, biodiversidad y hábitat. Se ha sugerido que la extracción de madera produce mayor alteración en el suelo en comparación con los efectos sobre la vegetación y la pérdida de especies a escala de ecosistema (Nasi y Frost, 2009).

La alteración de los ciclos de $\mathrm{C}$ y $\mathrm{N}$ se debe a la remoción de biomasa subterránea (raíces), esto disminuye la captura de C, de igual forma decrece la asignación subterránea de $\mathrm{C}$ y la biomasa microbiana del suelo acelerando el recambio de raíces y disminuyendo su masa, promoviendo la disponibilidad de amonio y nitratos y la volatilización de N (Nave, Vogel, Gough y Curtis, 2009). Además, se observa una aceleración de los procesos de mineralización de C, nitrificación y desnitrificación (Nave et al., 2009). Asimismo, Nordén, Penttilä, Siitonen, Tomppo y Ovaskainen. (2013) hallaron una reducción de hongos de 35\% incluso con una baja intensidad de cosecha.

En suma, es claro que las relaciones de interdependencia que ocurren en los bosques templados sugieren que la maximización de un determinado producto o servicio necesariamente impacta el nivel de producción de otros bienes y/o servicios ecosistémicos de los bosques, y desbalances en otros componentes y funciones del ecosistema. Este enfoque no ha sido abordado claramente en los bosques templados bajo aprovechamiento regulado en México, dejando la protección de los procesos y componentes ecosistémicos como objetivos complementarios y supeditados al aprovechamiento maderable, determinados por las presiones de incremento a la producción maderable nacional. Por ello, es imperativo integrar prácticas que ayuden a conservar la biodiversidad y los servicios ecosistémicos que proveen los bosques templados (Goldman et al., 2008; Raudsepp-Hearne, Peterson y Bennett, 2010).

\section{Las áreas naturales protegidas con bosques templados en México}

Los bosques templados están representados en 57 de las 176 ANP federales (32\%) (Fig. 2), de las cuales 11 son Reservas de la Biosfera, 30 Parques Nacionales, 13 áreas de protección de flora y fauna y tres área de protección de recursos naturales que en total ocupan una superficie de 7942843 ha (Tabla 3) (Rodríguez-
Bustos, 2013). La concepción más antigua de ANP estuvo orientada a un modelo proteccionista que separaba a la gente de los ecosistemas (Melo, 2002; West, Igoe y Brockington, 2006; Durand y Vázquez, 2011;) y las justificaciones para su decreto se basan en principios de protección de recursos hídricos y forestales. La primera ANP decretada en México en 1876 fue el Parque Nacional Desierto de los Leones; su estatus de protección estaba fundamentada en la belleza escénica de los paisajes, en el interés histórico del sitio como patrimonio de la nación y su importancia sobre la recarga de acuíferos a escala regional. A mitad del siglo XX las ANP pasaron de conservar paisajes y recursos naturales a concentrar los esfuerzos de conservación de especies y su hábitat (Palomo et al., 2014) (Fig. 2). Por ejemplo, el parque nacional Izta-Popo decretado en 1935 incluye ecosistemas por arriba de la cota de los $3600 \mathrm{msnm}$, protege principalmente a Pinus hartwegii por ser el hábitat del conejo teporingo Romerolagus diazi (Comisión Nacional de Áreas Naturales Protegidas [Conanp], 2013), excluyendo los bosques de Abies y Quercus que albergan el mayor aporte a la biodiversidad. A pesar de que algunos estudios cuestionan la efectividad de las ANP sobre la conservación de la biodiversidad, es importante señalar que fungen un papel imprescindible sobre el control de la deforestación de los bosques templados (Rodríguez-Bustos, 2013).

A pesar de las estrategias aplicadas para su conservación, las ANP en zonas de bosques templados no han escapado de un acelerado proceso de degradación ambiental dentro y fuera de sus límites (Rodríguez-Bustos y Galicia, 2016). Figueroa, SánchezCordero, Meave y Trejo. (2009) mencionan que las ANP en México tienen 55\% de Bosques Templados como área predominante, pero que solo $40 \%$ de esta superficie es efectiva para fines de conservación debido a que los límites de las ANP regularmente están sujetas a deforestación y cambio a usos de suelo urbano. Asimismo, se ha sugerido que los cambios más significativos y la extensión simplificada de especies en las ANP son ocasionados por un manejo. El establecimiento de un ANP instala nuevas reglas en torno al uso y manejo de los recursos naturales, modificando la relación de los habitantes con su entorno, así como la forma en que comprenden y construyen su espacio (Durand y Jiménez, 2010). 


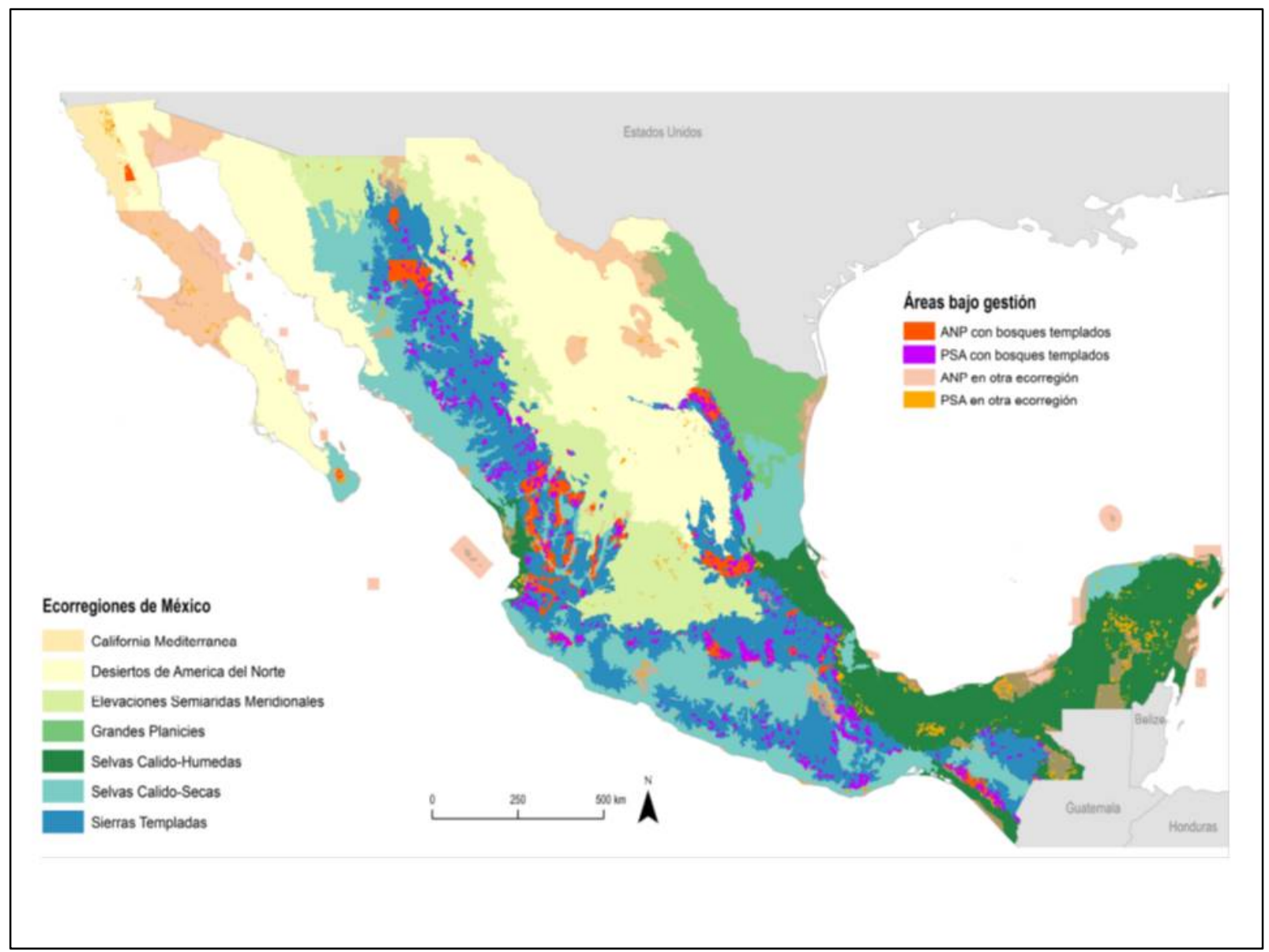

Figura 2. Mapa de la distribución de las Áreas Naturales Protegidas y los Programas de Pago de Servicios Ambientales en los bosques templados de México.

Merino y Martínez (2014) consideran el establecimiento de ANP como una de las estrategias de política pública que afecta el uso de recursos naturales, específicamente los forestales a escala comunitaria. Por ejemplo, hay registro de comunidades que no estuvieron informadas de restricciones para aprovechamientos forestales hasta que solicitaron permisos y fueron negados e incluso hay registro de suspensiones de actividades comunitarias. Debido a esto la conceptualización de las ANP ha cambiado en el tiempo y respondido al contexto político y cultural (Koleff y Urquiza-Haas, 2011).

El modelo proteccionista que excluye a los humanos de los ecosistemas (wilderness approach) (Durand y Jiménez, 2010; Palomo et al., 2014) evolucionó a un concepto de conservación que reconoce la complejidad social de las ANP (socio-ecological approach) (Durand y Vázquez, 2011; Koleff y Urquiza-Haas, 2011). A partir de entonces, se estableció el concepto de Reserva de Biosfera que se estableció bajo el objetivo de promover el manejo y la conservación de los ecosistemas, así como la investigación y la educación. Esta categoría de conservación es una muestra temprana del concepto socio-ecológico aplicada a la conservación porque incluye explícitamente a las poblaciones humanas y el uso sustentable de los recursos naturales como parte de las ANP (López-Medellín et al., 2017). La conservación solo puede ser efectiva si se integra la parte socioeconómica y política, para la construcción de capital social a través de redes de organización con la inclusión de los dueños de los bosques que permita identificar y atender conflictos sociales en torno al uso de los recursos forestales (Figueroa et al., 2011; López-Medellín et al., 2017). 
TABLA 3. Superficie de bosques templados bajo conservación gubernamental y en sistemas de protección independientes (Conabio, 2014).

\begin{tabular}{|c|c|c|c|c|c|c|c|c|}
\hline \multirow[b]{2}{*}{$\begin{array}{c}\text { Tipos de } \\
\text { Vegetación }\end{array}$} & \multicolumn{5}{|c|}{ Superficie de ANP (hectáreas) } & \multicolumn{3}{|c|}{ Superficie fuera de ANP (hectáreas) } \\
\hline & Federal & Estatal & Municipal & $\begin{array}{c}\text { Áreas } \\
\text { destinadas } \\
\text { voluntaria- } \\
\text { mente a la } \\
\text { conservación } \\
\end{array}$ & Total ANP & $\begin{array}{c}\text { Reservas } \\
\text { privadas } \\
\text { y sociales }\end{array}$ & $\begin{array}{c}\text { Total de } \\
\text { superficie } \\
\text { protegida }\end{array}$ & SINAP \\
\hline $\begin{array}{l}\text { Bosque de } \\
\text { coníferas }\end{array}$ & 1028656 & 257419 & 148 & 35762 & 1321985 & 15799 & 1337784 & 312557 \\
\hline $\begin{array}{l}\text { Bosque de } \\
\text { encinos }\end{array}$ & 998589 & 370381 & 5517 & 11398 & 1385885 & 85625 & 1471510 & 457067 \\
\hline Total & 2027245 & 627800 & 5665 & 47160 & 2707870 & 101424 & 2809294 & 769624 \\
\hline
\end{tabular}

Hoy en día, las ANP se han resignificado, pasando de ser simples fuentes de materia prima y escenarios donde solo importa la protección y conservación de la biodiversidad, a la oferta de beneficios intangibles como los servicios ecosistémicos culturales, reflejando nuevas vinculaciones entre sociedad y naturaleza. Por ejemplo, actualmente existe una fuerte tendencia a crear infraestructura y condiciones para atraer y fomentar el turismo en las ANP, de tal manera que la "conservación pasiva" pueda generar ingresos locales e incentive la conservación. No obstante, dadas las condiciones culturales y sociales que prevalecen en la mayoría de las ANP, es imperativo analizar detenidamente los enfoques, alcances e impactos de estos proyectos de ecoturismo, por las implicaciones que tienen en las relaciones locales, sobre todo en áreas que pueden ser muy sensibles por sus características socio ecológicas. Este es el caso de la reserva de la mariposa monarca, o de las barrancas del cobresierra Tarahumara, entre otros, donde los objetivos de desarrollo socioeconómico rebasan los objetivos de conservación biológica.

Para integrar esta nueva perspectiva socioecológica en las ANP es necesario considerar las causas de la problemática ambiental para poder incidir sobre el manejo para la protección y conservación, es decir, entender como los humanos (los diferentes actores) y nuestras instituciones somos los conductores del cambio. Por lo tanto, la inclusión de un marco de análisis socioecológico en los planes de protección y conservación es crucial por al menos tres factores: 1) proporcionaría mayor información acerca de la trayectoria de cambio del paisaje y sus conductores de cambio; 2) facilitaría identificar actividades productivas y tradicionales, de modo que los planes de manejo tendrían que ajustarse a los intereses de explotación comunitario; y 3) los planes de manejo deberían reconocer la importancia de conservar extensiones y especies específicas, pero también actividades y zonas de relevancia cultural con fines de conservación de patrimonio cultural, donde se incluyan prácticas y percepciones sociales sobre el uso de los recursos.

\section{Los bosques templados como proveedores de servicios ecosistémicos}

El concepto de servicios ecosistémicos ha sido ampliamente desarrollado a partir de un enfoque económico en las últimas décadas (Millennium Ecosystem Assessment [MEA], 2003; TEEB, 2010; Vihervaara, Rönkä y Walls 2010; Summers, Smith, Case, y Linthurst, 2012). En México, los bosques templados se han identificado como importantes proveedores de servicios ecosistémicos de aprovisionamiento (madera, alimentos, agua dulce, recursos químicos, recursos genéticos, bioenergía y recursos forestales no maderables); de regulación (clima y captura de carbono, filtrado de aire, estabilidad del suelo, control de deslizamientos de tierra): culturales (cohesión social y enraizamiento, recreación, educación), y servicios de apoyo al hábitat (diversidad biológica, productividad primaria, formación del suelo) (Balvanera y Cotler-Ávalos, 2007; Galicia y ZarcoArista, 2014). 
Los ecosistemas de bosque templado en México constituyen un capital natural asociado a un conjunto de servicios estratégicos a escala nacional, además, son fuente de productos de subsistencia de poblaciones suministrando ingresos económicos, estableciéndose así una relación entre el bienestar de la sociedad humana, la economía y los recursos forestales (Hinojosa-Flores, Skutsch y Mustalahti, 2016). La provisión de madera de especies arbóreas como los pinos y los encinos es uno de los servicios ecosistémicos más explotados en los bosques templados. De la producción del país, aproximadamente $70 \%$ se destina como madera aserrada, $18 \%$ para celulosa, $7 \%$ para combustible directo y carbón. Esto representa cerca de 93\% del valor total de la producción silvícola nacional (1336 millones de dólares), principalmente derivada de los bosques templados de México (Semarnat, 2011; Galicia y Zarco-Arista, 2014). Los bosques templados son grandes proveedores de agua porque son las cabeceras de cuencas en zonas altas, ya que están presentes en 77 de las 110 principales zonas de recarga y purificación de acuíferos en México (Arriaga, Aguilar y Alcocer, 2002). Además, estas zonas son también las de mayor concentración de población humana con más de 30 millones de habitantes y varias zonas metropolitanas, como la ciudad de México, Guadalajara y Puebla (Instituto Nacional de Estadística y Geografía [Inegi], 2010).

Por esta misma razón, tienen un efecto importante sobre la regulación del clima regional y de la calidad del aire; por ejemplo, en la Ciudad de México a través de los sistemas boscosos circundantes se fijan cada año cerca de $2 \%$ de las emisiones de $\mathrm{CO}_{2}, 2.3 \%$ de $\mathrm{O}_{3}$ y filtran $1.6 \%$ de contaminantes producidos, lo cual es suficiente para dar lugar a una mejora en la calidad del aire en la Ciudad de México (Chagoya y Gutiérrez, 2009). Los servicios de regulación pueden ser cruciales para abatir los costos económicos que generan los eventos meteorológicos extremos (inundaciones, erosión, etc.) (Ellis et al., 2015). Asimismo, estas disyuntivas tienen marcadas repercusiones económicas, debido a que la preponderancia de la extracción de madera reduce la diversificación económica de las comunidades que viven del bosque, aunque en los últimos años se ha propiciado la diversificación productiva del bosque, incluyendo el turismo (Ellis et al., 2015). Sin embargo, poco se conoce respecto a otros servicios de provisión, así como de soporte, regulación y culturales.
En México el pago por servicios ambientales (PSA) es un esquema que beneficia económicamente al sector forestal. El objetivo de los PSA es compensar a los dueños de los bosques a cambio de mantener la función proveedora de algunos procesos ecológicos que derivan en servicios ambientales específicos como la captación de lluvia y recarga de acuíferos (servicios hidrológicos), el mantenimiento de estructuras naturales que permitan el desarrollo de la fauna silvestre (hábitat para la biodiversidad) y la captura de carbono (Muñoz-Piña, Guevara, Torres y Braña, 2008; Chagoya y Gutiérrez, 2009). El funcionamiento del esquema considera la participación directa de las comunidades rurales, con acciones muy acotadas de conservación (zanjas y construcción de brechas cortafuego) y protección de partes altas de las cuencas, creación de estructuras para el refugio de la fauna silvestre y la reforestación con especies de rápido crecimiento (McAfee y Shapiro, 2010). Sin embargo, estas acciones implican cambios en algunos procesos del suelo (obras de captación de agua, acomodo de materiales) y de la vegetación (reforestaciones con especies no nativas o en lugares inadecuados) que pueden incidir negativamente en la integridad de los ecosistemas que pretenden proteger (Muñoz-Piña et al., 2008; McAfee y Shapiro, 2010). Estos aspectos no han sido evaluados en el marco del PSA, ni en los estudios del impacto del manejo de los bosques templados.

En 2007 (año de mayor extensión del programa) 2.6 millones de hectáreas de bosques templados en México estaban bajo algún tipo de PSA ( $2 \%$ de la superficie forestal nacional) con un presupuesto de alrededor de 450 millones de dólares (Costedoat et al., 2015) y escasos resultados en prevenir la deforestación (McAfee y Shapiro, 2010). De acuerdo con Semarnat, entidad emisora de las Reglas de Operación del Programa Nacional Forestal en las que se encuentran los lineamientos generales del PSA, el esquema de pagos está basado en áreas de pago diferenciado. Por ejemplo, MXN \$1100/ha/año y MXN \$382/ha/año para superficie mínima y máxima, respectivamente, más un ingreso extra por la asistencia técnica anual, lo que conlleva a cumplir ciertas obligaciones de las personas beneficiarias. El pago por servicios de conservación de la biodiversidad también está en un esquema diferenciado por áreas entre: MXN \$550/ha/año y MXN \$280/ha/año. Otros servicios como la conservación y restauración de suelos se pagan 
a MXN \$3100/ha con un adicional de MXN \$350/ha si se realizan de actividades en cárcavas; la reforestación está tabulada en MXN \$1772/ha, mientras que la protección de áreas reforestadas fluctúa entre MXN \$2514/ha y MXN \$1320/ha. Existen también otras instancias gubernamentales federales que apoyan el PSA, sobre de esto la Comisión Nacional Forestal [Conafor] en el periodo 2003-2008 generó un presupuesto de 2929.025 millones de pesos mexicanos, distribuidos de la siguiente manera: hidrológicos 2414.69 millones de pesos; conservación de la biodiversidad 361.72 millones de pesos; sistemas agroforestales 138.8 millones de pesoso; fijación carbono 0.715 millones de pesos; y fomento a la regeneración natural 13.1 millones de pesos.

El impacto del programa no es muy claro, ya que el PSA de los bosques templados proviene de programas gubernamentales, es decir, no existe aún un mercado definido en la región central de México que promueva la oferta y demanda de estos servicios ecosistémicos. Torres y Guevara (2002) consideran que entre los factores que limitan una producción sostenida de servicios ecosistémicos en México están la ausencia de un mercado definido y la escasez de información sobre su cuantía y las relaciones con los procesos ecosistémicos que las generan. Otro problema subyacente del PSA es la falta de sustento técnicocientífico que justifique y dimensiones la provisión de los servicios en función de las características de los ecosistemas, lo cual limita el número de alternativas de manejo que aseguren la sustentabilidad de los mismos (Torres y Guevara, 2002). El PSA hidrológicos es un ejemplo claro de la importancia diferencial de las características ecológicas (composición vegetal, clima, geomorfología, etc.), en la captación de lluvia (Torres y Guevara, 2002), lo cual no se considera en los esquemas de pagos del programa. Las autoridades federales consideran que el programa es exitoso y una alternativa a la pobreza, mientras que las localidades consideran que los pagos son insuficientes, que deben mejorarse debido al alto costo de oportunidades de conservar áreas forestales. Por ejemplo, el pago a cinco años no permite una gestión forestal adecuada que necesita un horizonte de mínimo 20 años (Fuentes-Pangtay, 2009; Perevochtchikova y Oggioni, 2013; Almeida et al., 2014).

Lo anterior sugiere que el PSA espera pasivamente favorecer la permanencia del esquema a través del desarrollo de un mercado definido, e incrementar la cantidad de áreas bajo este programa. Es decir, el enfoque socioecológico en este programa aun esta desvinculado, ya que los dueños no dimensionan la importancia de los servicios ambientales que sus bosques proveen, sino el ingreso fijo que reciben por realizar actividades previamente definidas y acotadas, independientemente de las características de sus recursos forestales. Esta es una gran diferencia con el manejo forestal con fines de producción maderable. Adicionalmente, la efectividad de los programas de PSA debe de ser calificada a través del monitoreo y de evaluaciones ecológicas y sociales, así como reconocer la percepción de las autoridades y comunidades locales sobre los efectos, beneficios y expectativas del PSA (Perevochtchikova y Oggioni, 2013; Costedoat et al., 2015). No obstante, las investigaciones para dimensionar y valorar los servicios ambientales, así como el monitoreo de su desempeño implican altos costos que el programa no ha podido asumir, y se mantiene la inercia del pago fijo independiente de las características particulares de cada caso.

\section{El enfoque socioecológico}

La aplicación del enfoque socioecológico en los bosques templados es clave para el mantenimiento, el uso sostenible y la resiliencia de los bosques; y para asegurar el uso de bienes tangibles e intangibles, y el ingreso de las comunidades forestales. En México, los bosques y la gente que vive de ellos están estrechamente unidas y funcionan dentro de sistemas adaptativos complejos (las relaciones y retroalimentaciones entre las estructuras y procesos ecológicos con las estructuras y procesos sociales, e instituciones están intrínsecamente vinculados) (Fig. 3). Las necesidades sociales a través de los usos consuntivos (servicios ecosistémicos de provisión) y no consuntivos (servicios ecosistémicos culturales) y las elecciones de las prácticas de manejo afectan los procesos ecosistémicos que brindan los servicios ecosistémicos, su flujo y distribución, por lo cual deben ser entendidos e incorporados en las decisiones de gobierno, elucidando efectos de las decisiones de manejo en ambos componentes del socioecosistema (Fig. 3). Asimismo, es necesario desarrollar nuevas alternativas en la toma de decisiones de manejo y gobernanza para promover acciones humanas que permitan el uso sostenible en estos ecosistemas (Fig. 3) (Bürgi y Gimmi, 2007). La aplicación del concepto de servicios 
ecosistémicos en un marco de socio-ecosistemas permite identificar las interacciones socioecológicas e integrarlas en la toma de decisiones aplicadas al manejo forestal, las ANP y el PSA en los bosques templados de México; subrayando la importancia de regular y fortalecer el sistema de control y seguimiento de los actores sociales y sus actividades.

En México, el principal uso consuntivo de los bosques templados es a través del aprovechamiento forestal, que es la forma más elaborada de interacción entre las comunidades con los ecosistemas. Con el tiempo esta relación ha evolucionado hacia una integración de las comunidades con los bosques en un contexto de mercado. Sin embargo, el interés de los propietarios por el aprovechamiento de sus recursos maderables ha ido más allá de la iniciativa gubernamental de incrementar la oferta nacional de madera, a un papel más activo de los productores en la búsqueda de oportunidades para el aprovechamiento de otros productos no maderables, o el pago por servicios ecosistémicos. Si bien es cierto que internacionalmente no ha presenta resultados unívocos, en el caso mexicano parece ser una herramienta útil para la construcción de sustentabilidad (Fig. 3) (SeguraWarnholtz, 2014), principalmente porque las comunidades recuperan el sentimiento de propiedad sobre sus recursos, a pesar de los problemas de inequidad al interior de las propias comunidades (Vázquez, 2015; Perevochtchikova, 2016). Reconocer la historia de uso de los recursos forestales y sus impactos sobre los ecosistemas forestales es una manera de identificar efectivamente cuáles han sido los efectos positivos y negativos del manejo de recursos forestales (Fig. 3).

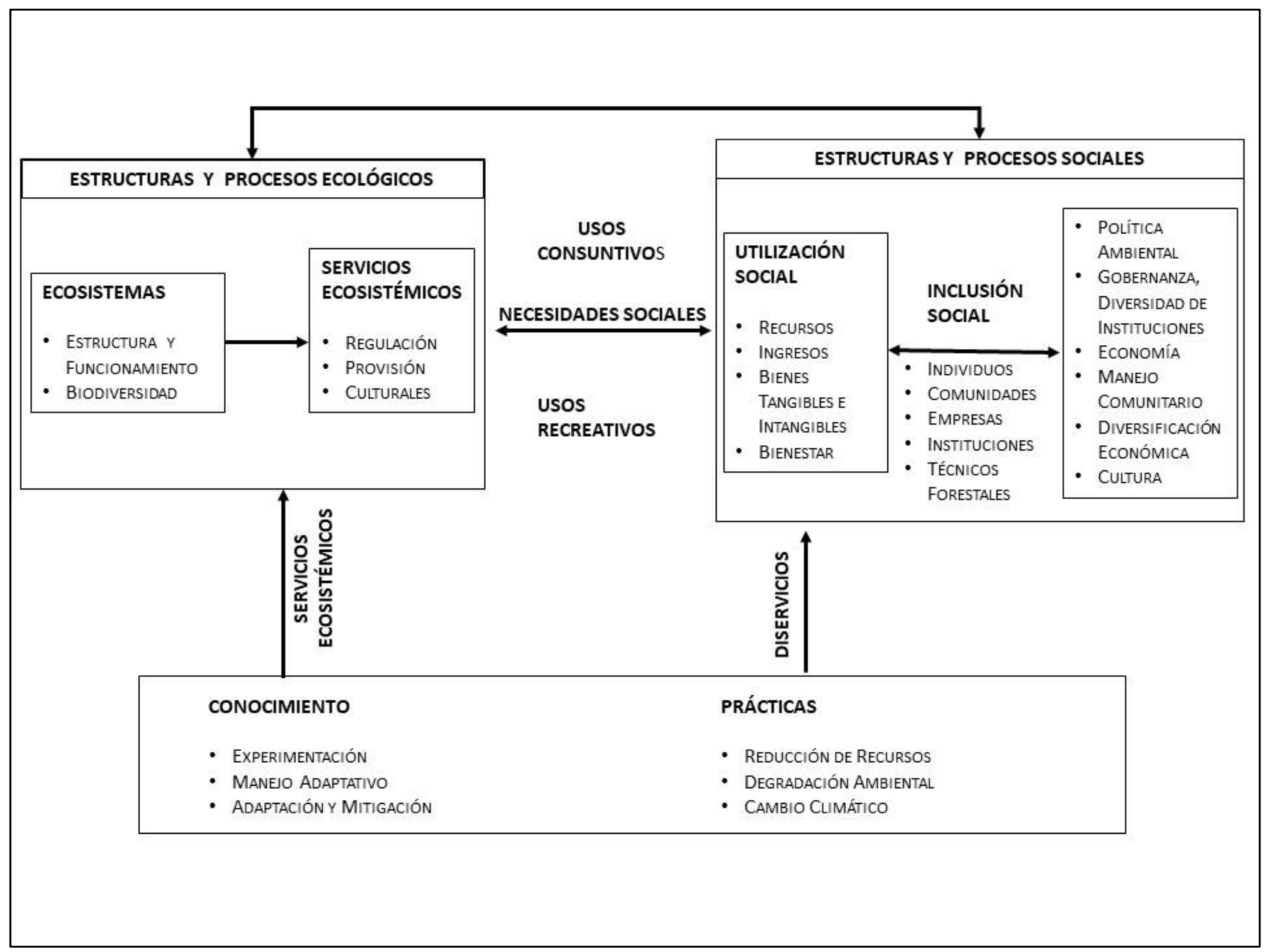

FIGURA 3. Propuesta de la integración del enfoque socio-ecológico de los bosques templados de México. 
La implementación de instrumentos de política pública, como las ANP y el PSA, requieren del establecimiento de las interacciones entre los diferentes elementos biofísicos, sociales y los instrumentos económicos y políticos que conforman un sistema socio-ecológico. Los servicios ecosistémicos deben tener la capacidad de demostrar la relación causal entre el cambio en un atributo del ecosistema y una medida resultante de bienestar humano o social. Por ejemplo, es vital reconocer cómo las decisiones de manejo, las ANP o el PSA pueden contribuir al aprovisionamiento de servicios ecosistémicos, como el secuestro de carbono en suelos para la mitigación del clima, entre otros.

Existe un consenso en la relevancia de la valuación económica de los servicios ecosistémicos como instrumento importante en la toma de decisiones de la importancia de los ecosistemas y de los servicios que proveen para el bienestar de las sociedades (Costanza et al., 1997; De Groot, Wilson y Boumans, 2002; Daily et al., 2009). Una alternativa es la posibilidad de utilidades directas de los bienes y servicios para los pobladores y la implementación de incentivos locales para la conservación. Para esto es necesario establecer mecanismos de gobernanza para toma de decisiones incluyentes y esquemas para el desarrollo de capacidades locales (Fig. 3). En este sentido, el enfoque socioecológico favorece la transición de un esquema de exclusión de la parte social a uno de participación e inclusión de las comunidades locales y otros múltiples actores (Fig. 3).

Finalmente, las ANP se han resignificado, pasando de ser simples fuentes de materia prima y escenarios donde solo importa la protección y conservación de la biodiversidad, a la oferta de beneficios intangibles como los servicios ecosistémicos culturales, reflejando nuevas vinculaciones entre sociedad y naturaleza (Fig. 3). Sin embargo, pocos estudios en relación con las áreas protegidas se han centrado en variables ecológicas y socioculturales en estos territorios ecológicamente frágiles, para orientar planes y programas encaminados a lograr tanto la conservación de los recursos naturales como el uso y disfrute de los recursos (Fig. 3).

\section{RECONOCIMIENTOS}

El primer autor agradece a la Dirección General de Asuntos del Personal Académico-Programa de Apoyo a Proyectos de Investigación e Innovación Tecnológica (DGAPA-PAPIIT,
UNAM) al proyecto: "Influencia de la química, morfología y área foliar de tres especies arbóreas sobre la productividad, descomposición y respiración del suelo en bosques templados del centro de México", IN104515 por el apoyo para realizar esta revisión. Asimismo, el primer autor agradece a Alba Esmeralda Zarco Arista por el apoyo en la elaboración y diseño de las figuras.

\section{REFERENCIAS}

Acosta-Mireles, M., Carrillo-Anzures, F., \& Díaz Lavariega, M. (2009). Determinación del carbono total en bosques mixtos de Pinus patula. Terra Latinoamericana, 27(2), 105-114.

Adcharaporn, P., Kim, Y. S., \& Daughtery, P. J. (2006). What makes community forest management successful: A meta-study from community forest through the world. Society and Natural Resources, 19(1), 33-52. doi: 10.1080/08941920500323260

Aguirre-Planter, E., Furnier, G. R., \& Eguiarte, L. E. (2000). Low levels of genetic variation within and high levels of genetic differentiation among populations of species of Abies from southern México and Guatemala. American Journal of Botany, 87, 362-371. doi:10.2307/2656632.

Almeida-Leñero, L., Revollo-Fernandez, D., Caro-Borrero, A., Figueroa, F., Espinosa, M.D., Cruz, G., \& Mazari-Hiriart, M. (2014). El Pago por Servicios Ambientales en México 2010: una evaluación multidisciplinaria. En Pago Por Servicios Ambientales en México. Un Acercamiento para su Estudio (pp. 155-180). El Colegio de México, México.

Álvarez-Arteaga, G., García Calderón, N., Krasilnikov, P., \& GarcíaOliva, F. (2013). Almacenes de carbono en bosques montanos de niebla de la sierra norte de Oaxaca, México. Agrociencia, 47(2), 171180.

Antinori, C., \& Rausser, G. (2007). Collective Choice and Community Forestry Management in Mexico: An Empirical Analysis. Journal of Development Studies, 43(3): 512-536. doi: 10.1080/00220380701204471

Arriaga L, Aguilar V, \& Alcocer J. (2002). Aguas continentales y diversidad biológica de México. México, D.F.: Comisión Nacional para el Conocimiento y Uso de la Biodiversidad.

Augusto, L., De Schrijver, A., Vesterdal, L., Smolander, A., Prescott, C., \& Ranger, J. (2014). Influences of evergreen gymnosperm and deciduous angiosperm tree species on the functioning of temperate and boreal forests. Biological Reviews, 90(2):444-466. doi: 10.1111/brv.12119.

Balvanera, P., \& Cotler-Ávalos, H. (2007). Acercamientos al estudio de los servicios ecosistémicos. Gaceta Ecológica, 84, 8-15.

Binkley, D. (1997). Bioassays of the influence of Eucalyptus saligna and Albizia falcataria on soil nutrient supply and limitation. Forest 
Ecology and Management, 91, 229-234. doi: 10.1016/S03781127(96)03861-3

Boiffin, J., \& Munson, A. D. (2013). Three large fire years threaten resilience of closed crown black spruce forests in eastern Canada. Ecosphere, 4(5), 56. doi: 10.1890/ES13-00038.1

Bowler, D. E., Buyung-Ali, L., Healey, J. R., Jones, J. PG., Knight, T. M., \& Pullin, A. S. (2012). Does community forest management provide global environmental benefits and improve local welfare? Frontiers in Ecology and Environment 10(1), 29-36. doi: $10.1890 / 110040$

Bray, D., Merino, L., \& Barry, D. (eds). (2007). Los bosques comunitarios de México: Manejo sustentable de paisajes forestales. México: Instituto Nacional de Ecología (INE-Semarnat), Consejo Civil Mexicano para la Silvicultura Sostenible, Instituto de Geografía, UNAM, Florida International Institute.

Bruelheide H., \& Luginbühl, U. (2009). Peeking at ecosystem stability: making use of a natural disturbance experiment to analyze resistance and resilience. Ecology, 90(5), 1314-25. doi: 10.1890/072148.1

Bürgi M, \& Gimmi U. (2007). Three objectives of historical ecology: the case of litter collecting in Central European forests. Landscape Ecology, 22, 77-87.

Caballero-Deloya., M. (2008). El “otro" México forestal (La actividad forestal ilícita). Revista Ciencias Forestales en México, 33(103), 149-175

Cárdenas, I. (2008). Efecto de la calidad del tejido foliar sobre el proceso de descomposición en bosques de Pino-Encino, bajo condiciones contrastantes de precipitación (tesis de maestría). Universidad Nacional Autónoma de México, México.

Cantú-Silva, I., Sánchez-Castillo, L., González-rodríguez, H., Kubota, T., Gómez-Meza, v., \& Domínguez-Gómez, G. (2013). Retorno potencial de nutrimentos por componentes secundarios de la hojarasca en comunidades vegetales de Nuevo León. Revista Mexicana de Ciencias Forestales, 4(17), 138-155.

Chagoya, J, \& Gutiérrez, L. (2009). Esquema de pago por servicios ambientales de la Comisión Nacional Forestal, México. Turrialba, Costa Rica: CATIE.

Challenger, A. (1998). Utilización y conservación de los ecosistemas terrestres de México: pasado, presente y futuro. Ciudad de México, México: Comisión Nacional para el Uso y Conocimiento de la Biodiversidad, Instituto de Biología de la UNAM y Agrupación Sierra Madre S.C.

Chávez-Vergara, B. M. (2010). Efecto de dos especies del género Quercus sobre la dinámica de C, N y P en un fragmento forestal de la cuenca de Cuitzeo, Michoacán (tesis de maestría). Universidad Nacional Autónoma de México, México.

Chávez-Vergara, B., González-Rodríguez, A., Etchevers, J. D., Oyama, K., \& García-Oliva, F. (2015). Foliar nutrient resorption constrains soil nutrient transformations under two native oak species in a temperate deciduous forest in Mexico. European Journal of Forest Research, 134(5), 803-817. doi: 10.1007/s10342015-0891-1

Comisión Nacional para el Conocimiento y Uso de la Biodiversidad [Conabio]. (2001). Estrategia nacional sobre biodiversidad de México. Ciudad de México, México: Comisión Nacional para el Conocimiento y Uso de la Biodiversidad.

Comisión Nacional para el Conocimiento y Uso de la Biodiversidad [Conabio] (2014). Bosques mesófilos de montaña de México: diversidad, ecología y manejo. Ciudad de México, México: Comisión Nacional para el Conocimiento y Uso de la Biodiversidad.

Comisión Nacional de Áreas Naturales Protegidas [Conanp]. (2014). Cobertura de las Areas Naturales Protegidas Federales de México y áreas destinadas voluntariamente a la conservación certificadas por la Comisión Nacional de Áreas Protegidas de México. México: Conanp.

Costanza, R., dArge, R., de Groot, R., Farber, S., Grasso, M., Hannon, B., Limburg, K., Naeem, S., Oneill, R.V., Paruelo, J., Raskin, R.G., Sutton, P., \& van den Belt, M. (1997). The value of the world's ecosystem services and natural capital. Nature, 387, 253-260. doi:10.1038/387253a0

Costedoat, S., Corbera, E., Ezzine-de-Blas, D., Honey-Rosés, J., Baylis, K., \& Castillo-Santiago, M.A. (2015). How Effective Are Biodiversity Conservation Payments in Mexico? PLOS ONE, 10, e0119881. doi:10.1371/journal.pone.0119881

Cronkleton, P., Bray, D. B., \& Medina, G. (2011). Community Forest Management and the Emergence of Multi-Scale Governance Institutions: Lessons for REDD plus Development from Mexico, Brazil and Bolivia. Forests, 2(2), 451-473. doi: 10.3390/f2020451

Cruz-Flores, G., \& Etchevers-Barra, J. D. (2011). Contenidos de carbono orgánico de suelos someros en pinares y abetales de áreas protegidas de México. Agrociencia, 45(8), 849-862.

Daily, G. C., Polasky, S., Goldstein, J., Kareiva, P., Mooney, H. A., Pejchar, L., Ricketts, T.H. Salzman, J., \& Shallenberger, R. (2009). Ecosystem services in decision-making: time to deliver. Frontiers in Ecology and the Environment, 7, 21-28. 10.1890/080025

De Groot, R.S., Wilson, M., Boumans, R. (2002). A typology for the description, classification and valuation of ecosystems functions, goods and services. Ecological Economics, 41 (3), 393.408.

De Jong, B., Cairns, M., Haggerty, P., Ramirez-Marcial, N., OchoaGaona, S., Mendoza-Vega, J., \& March-Mifsut, I. (1999). Landuse change and carbon flux between 1970s and 1990s in central highlands of Chiapas, Mexico. Environmental Management, 23(3), 373-385. doi: $10.1007 / \mathrm{s} 002679900193$

Delgadillo, E. (2011). Productividad primaria neta de los bosques templados de la cuenca del río Magdalena (tesis de maestría). Universidad Nacional Autónoma de México, México.

Delgado, P., Piñero, D., Chaos, A., Pérez-Nasser, N., \& Alvarez-Buylla, E. (1999). High levels of variation and structure genetic in very 
rare pine species (Pinus rzedowskii), endemic of Michoacán, México. American Journal of Botany, 86, 669-676. doi:10.2307/2656576.

Durand, L., \& Jiménez, J. (2010). Sobre áreas naturales protegidas y la construcción de no-lugares. Notas para México. Revista Lider, 16(12), 59-72.

Durand, L., \& Vázquez, L. B. (2011). Biodiversity conservation discourses. A case study on scientists and government authorities in Sierra de Huautla Biosphere Reserve, Mexico. Land Use Policy, 28(1), 76-82. doi: 10.1016/jlandusepol.2010.04.009

Ellis, E. A., Kainer, K. A., Sierra-Huelsz, J. A., Negreros-Castillo, P., Rodriguez-Ward, D., \& DiGiano, M. (2015). Endurance and Adaptation of Community Forest Management in Quintana Roo, Mexico. Forests, 6(11), 4295-4327. doi:10.3390/f6114295

Organización de las Naciones Unidas para la Alimentación y la Agricultura [FAO]. (2015). La Evaluación de los recursos forestales mundiales. Roma, Italia.

Farjon, A. \& Styles, B. T. (1997). Pinus (Pinaceae). Flora Neotropica Monograph 75. Bronx, NY: The New York Botanical Garden.

Figueroa, F., Sánchez-Cordero, V., Meave, J. A., \& Trejo, I. (2009) Socioeconomic context of land use and land cover in Mexican biosphere reserves. Environmental Conservation, 36, 180-191. doi: $10.1017 /$ S0376892909990221

Figueroa, F., Sánchez-Cordero, V., Illoldi-Rangel, P., \& Inaje, M. (2011). Evaluación de la efectividad de las áreas protegidas para contener procesos de cambio en el uso del suelo y la vegetación. ¿Un índice es suficiente?. Revista Mexicana de Biodiversidad, 82(3), 951-963.

Finér, L., Ohashi, M., Noguchi, K., \& Hirano, Y. (2011). Factors causing variation in fine root biomass in forest ecosystems. Forest Ecology and Management, 261(2), 265-277. doi: 10.1016/j.foreco.2010.10.016

Finér, L., Helmisaari, S., Lõhmus, K., Majdi, H., Brunner, I., Børja, I., \& Vanguelova, E. (2007). Variation in fine root biomass of three European tree species: Beech (Fagus sylvatica L.), Norway spruce (Picea abies L. Karst.), and Scots pine (Pinus sylvestris L.). Plant Biosystems, 141(3), 394-405. doi: 10.1080/112663500701625897

Fuentes-Pangtay, T. (2009). Análisis de los programas de pago o compensación por servicios ambientales en la cuenca del Pixiquiac: fortalezas y debilidades en el contexto local. Documento técnico. Proyecto: NCMA3-08-03. Sendas, A.C.

Fürstenau, C., Badek, F. W., Lasch, P., Lexer, M. J., Linder, M., Mohr, P., \& Suckow, F. (2007). Multiple-use forest management in consideration of climate change and the interests of stakeholder groups. European Journal of Forest Research, 126, 225-239. doi: 10.1007/s10342-006-0114-x

Galicia, L., Potvin, C., \& Messier, Ch. (2015). Maintaining the high diversity of pine and oak species in Mexican temperate forests: a new management approach combining functional zoning and ecosystem adaptability. Canadian Journal of Forest Research, 45(10), 1358-1368. doi: 10.1139/cjfr-2014-0561

Galicia, L., \& Zarco-Arista, A. E. (2014). Multiple ecosystem services, possible trade-offs and synergies in a temperate forest ecosystem in Mexico: a review. International Journal of Biodiversity Science, Ecosystem Services and Management, 10(4), 275-288. doi: $10.1080 / 21513732.2014 .973907$

García-Montiel, C., \& Binkley, D. (1998). Effect of Eucalyptus saligna and Albiz̨ia falcataria on soil processes and nitrogen supply in Hawaii. Oecologia, 113(4), 547-556. doi: 10.1007/s004420050408

García-Oliva, F., Covaleda, S., Gallardo, J., Prat, Ch., Velázquez-Durán, R., \& Etchevers, J. (2014). Firewood extraction affects carbon pools and nutrients in remnant fragments of temperate forests at the Mexican Transvolcanic Belt. Bosque, 35(3), 311-324. doi: 10.4067/S0717-92002014000300006

Goldman, R. L., Tallis, H., Kareiva, P., \& Daily, G. C. (2008). Field evidence that ecosystem service projects support biodiversity and diversify options. Proceedings of the National Academy of Sciences of the United States of America, 105, 9445-9448. doi: $10.1073 /$ pnas.0800208105

Gómez-Pompa, A. 1985. Reflexiones Sobre los Recursos Bióticos de México. México: Alhambra.

Grier, C. C., Vogt, K., Keyes, M., \& Edmonds, R. L. (1981). Biomass distribution and above- and below-ground production in young and mature Abies amabilis zone ecosystems of the Washington Cascades. Canadian Journal of Forest Research, 11(1), 155-167. doi: $10.1139 / \mathrm{x} 81-021$

Harvey, B. J., \& Holzman, B. A. (2014). Divergent successional pathways of stand development following fire in a California closed-cone pine forest. Journal of Vegetation Science, 25, 89-99. doi: $10.1111 /$ jvs. 12073

Flores, I. D., Skutsch, M., \& Mustalahti, I. (2016). Impacts of Finnish cooperation in the Mexican policy making process: From the community forest management to the liberalization of forest services. Forest Policy and Economics, 73, 229-238. doi: 10.1016/j.forpol.2016.09.011

Hoekstra, J. M., Boucher, T.M., Ricketts, T. H., \& Roberts, C. (2005). Confronting a biome crisis: global disparities of habitat loss and protection. Ecology Letters, 8, 23-29. doi: 10.1111/j.14610248.2004.00686.x

Hooper, D., Chapin, S., Ewel, J., Hector, A., Inchausti, P., Lavorel, S., Lawton, J., Lodge, D., Loreau, M., Naeem, S., Schmid, B., Symstad, A., Vandermeer, J., \& Wardle D. (2005). Effects of biodiversity on ecosystem functioning: a consensus of current knowledge. Ecological Monographs, 75, 3-35. doi: 10.1890/04-0922

Instituto Nacional de Estadística y Geografía [Inegi]. (2010). Localidades de la República Mexicana, 2010, escala: 1:1. Obtenido de Principales resultados por localidad (ITER). Censo de Población 
y Vivienda 2010. Ciudad de México, México: Comisión Nacional para el Conocimiento y Uso de la Biodiversidad.

Kolb, M., \& Galicia, L. (2012). Challenging the linear forestation narrative in the Neo-tropic: regional patterns and processes of deforestation and regeneration in southern Mexico. The Geographical Journal, 178(2), 147-161. doi: 10.1111/j.14754959.2011.00431.x

Koleff, P., Urquiza-Haas, T. (Coords). (2011). Planeación para la conservación de la biodiversidad terrestre en México: retos en un país megadiverso. México: Comisión Nacional para el Conocimiento y Uso de la Biodiversidad-Comisión Nacional de Áreas Naturales Protegidas.

López-López, M. A., Velázquez-Martínez, A., \& Acosta-Montoya, J. (2014). Biomass distribution in declining sacred-fir seedlings. Interciencia, 31(6), 420-455.

López-Medellín, X., Vázquez, L. B., Valenzuela-Galván, D., Wehncke, E., Maldonado-Almanza, B., \& Durand-Smith, L. (2017). Percepciones de los habitantes de la Reserva de la Biósfera Sierra de Huautla: hacia el desarrollo de nuevas estrategias de manejo participativo. Interciencia, 42(1), 8-16. doi: 0378-1844/14/07/46808

Manzano M., D., López L., M. A., Manzano M., F., \& Pineda H., E. (2007). Productividad primaria neta de una plantación de recuperación de suelos. Morelia, México: VIII Congreso Mexicano de Recursos Forestales. Memorias en extenso.

Masera, O., Bellon, M., \& Segura, G. (1995). Forest management options for sequestering carbon in Mexico. Biomass and Bioenergy, 8(5), $357-$ 367. doi: 10.1016/0961-9534(95)00028-3

Masera, O. R., Ordoñez, M. J., \& Dirzo, R. (1997a). Carbon emissions from Mexican forests: current situation and long-term scenarios. Climatic change, 35(3), 265-295. doi: 10.1023/A:1005309908420

Masera, O., Bellon, M. R., \& Segura, G. (1997b). Forestry options for sequestering carbon in Mexico: comparative economic analysis of three case studies. Critical Reviews in Environmental Science and Technology, 27(S1), 227-244. doi: 10.1080/10643389709388522

Masera, O. R., Ceron, A. D., \& Ordóñez, J. A. (2001). Forestry mitigation options for México: Finding synergies between national sustainable development priorities and global concerns. Mitigation and Adaptation Strategies for Climate Change, 6(3-4), 289-310. doi: 10.1023/A:10133270.

McAfee, K., \& Shapiro, E. (2010). Payments for ecosystem services in Mexico: nature, neoliberalism, social movements, and the State. Nature and Society, 100(3), 579-599. doi: 10.1080/00045601003794833

Millennium Ecosystem Assessment [MEA]. (2003). Ecosystems and human well-being: a framework for assessment. Washington, Estados Unidos: Island Press.
Melillo, J., Aber J. D., \& Muratore, J. (1982). Nitrogen and lignin control of hardwood leaf litter decomposition dynamics. Ecology, 63(3), 621-626. doi: 10.2307/1936780

Melo, C. (2002). Áreas Naturales Protegidas de México en el Siglo XX. México: Instituto de Geografía, Universidad Nacional Autónoma de México.

Mendoza-Ponce, A., \& Galicia, L. (2010). Aboveground and belowground biomass and carbon pools in Highland temperate forest landscape in Central Mexico. Forestry, 83(5), 497-506. doi: $10.1093 /$ forestry/cpq032

Merino, L., Martínez, A.E. (2014). A vuelo de pájaro: Las condiciones de las comunidades con Bosques Templados en México. CONABIO. México.

Muñoz-Piña, C., Guevara, A., Torres, J. M., \& Braña, J. (2008). Paying for the hydrological services of Mexico's forests: analysis, negotiations and results. Ecological Economics, 65(4), 725-736. doi: 10.1016/j.ecolecon.2007.07.031

Návar-Cháidez, J. J., \& Jurado-Ybarra, E. (2009). Productividad foliar y radicular en ecosistemas forestales del Noreste de México. Revista Ciencia Forestal en México, 34, 89-106.

Nasi, R., \& Frost, P. G. H. (2009). Sustainable forest management in the tropics: is everything in order but the patient still dying? Ecology and Society, 14(2), 40. Recuperado de http://www.ecologyandsociety.org/vol14/iss2/art40/

Nave, L. E., Vogel, C. S., Gough, C. M., \& Curtis, P. S. (2009). Contribution of atmospheric nitrogen deposition to net primary productivity in a northern hardwood forest. Canadian Journal of Forest Research, 39, 1108-1118. doi: 10.1139/b11-026

Negreros, P., \& Snook, L. K. (1984). Análisis del efecto de la intensidad de corta sobre la regeneración de pinos en un bosque mezclado de pino-encino. Ciencia Forestal, 47, 48-61.

Nixon, K.C. (1993). The genus Quercus in Mexico. En Ramamoorthy, T. P., Bye R., Lot, A., \& Fa, J. (Eds), Biological diversity of Mexico: origins and distribution (pp. 447-458). Nueva York, Estados Unidos: Oxford University Press.

Diario Oficial de la Federación [DOF]. (2010). NORMA Oficial Mexicana NOM-059-SEMARNAT-2010, Protección ambiental-Especies nativas de México de flora y fauna silvestres-Categorias de riesgo y especificaciones para su inclusión, exclusión o cambio-Lista de especies en riesgo.

Nordén, J., Penttilä, R. Siitonen, J. Tomppo, E., \& Ovaskainen, O. (2013). Specialist species of wood-inhabiting fungi struggle while generalists thrive in fragmented boreal forests. Journal of Ecology, 101, 701-712. doi: 10.1111/1365-2745.12085

Oostra, H., Majdi, H., \& Olsson, M. (2006). Impact of tree species on soil carbon stocks and soil acidity in southern Sweden. Scandinavian Journal of Forest Research, 21, 364-371. doi: $10.1080 / 02827580600950172$

Ordóñez, J., De Jong, B., García-Oliva, F., Aviña, F., Pérez, J., Guerrero, G., \& Masera, O. (2008). Carbon content in vegetation, litter, and 
soil under 10 different land-use and land-cover classes in the Central Highlands of Michoacan, Mexico. Forest Ecology and Management, 255(7), 2074-2084. doi: 10.1016/j.foreco.2007.12.024

Palacio-Prieto, J. L., Bocco, G., Velázquez, A., Mas, J. F., Takaki-Takaki, F., Victoria, A., \& Trejo-Vázquez, I. (2000). La condición actual de los recursos forestales en México: resultados del Inventario Forestal Nacional 2000. Investigaciones geográficas, (43), 183-203.

Palomo, I., Montes, C., Martín-López, B., González, J. A., GarcíaLlorente, M., Alcorlo, P., \& García, M. R. (2014). Incorporating the social-ccological approach in protected areas in the anthropocene. BioScience, 64(3), 1-11. doi: 10.1093/biosci/bit033

Perevochtchikova, M. (2016). Estudio de los efectos del Programa de Pago por Servicios Ambeintales. Experiencia en Ajusco. Ciudad de México, México: El Colegio de México.

Perevochtchikova, M., \& Oggioni, J. (2013). Global and Mexican analytical review of the state of the art on Ecosystem and Environmental services: A geographical approach. Investigaciones Geográficas Boletín del Instituto de Geografia, 85, 47-65. doi: 10.14350/ rig.41239

Pérez-Suárez, M., Arredondo-Moreno, J., Huber-Sannwald, E., \& Vargas-Hernández, J. (2009). Production and quality of senesced and green litterfall in a pine-oak forest in central-northwest Mexico. Forest ecology and Management, 258(7), 1307-1315. doi: 10.1016/j.foreco.2009.06.031

Perry, J. P. (1991). The pines of Mexico and Central America. Portland, OR: Timber Press.

Pineda-López, M., Sánchez-Velázquez, L., Vázquez-Domínguez, G., \& Rojo, A. (2013). The effects on land use change on carbon content in the aerial biomass of an Abies religiosa forest in central Veracruz, Mexico. Forest Ecosystems, 22(1), 82-93.

Raudsepp-Hearne, C., Peterson, G. D., \& Bennett, E. M. (2010). Ecosystem service bundles for analyzing tradeoffs in diverse landscapes. Proceedings of Natural Academy of Science, 107, 5242-5247. doi: $10.1073 /$ pnas.0907284107

Reyes-Carrera, S. A., Méndez-González, J. Nájera-Luna, J. A., \& CeranoParedes, J. (2012). Producción de hojarasca en un rodal de Pinus cembroides en Arteaga, Coahuila, México y su relación con las variables climáticas. Revista Chapingo Serie Ciencias Forestales y del Ambiente, 19(1): 147-155.

Rocha-Loredo, A., \& Ramírez-Marcial, N. (2009). Producción y descomposición de hojarasca en diferentes condiciones sucesionales del bosque de pino-encino en Chiapas, México. Boletín de la Sociedad Botánica de México, (84), 1-12.

Rodríguez-Bustos. L. (2013). Transformación del paisaje en la zona centro de la región Izta-Popo (1980-2013) (Tesis de licenciatura). Universidad Veracruzana, Xalapa, Veracruz, México.

Rodríguez-Bustos, L., \& Galicia, L. (2016). Causas locales de la transformación del paisaje en una región montana del centro de
México. Acta Universitaria, 26(6), 83-94 http://dx.doi.org/10.15174/au.2016.1168

Ruiz-Aquino, F., Valdez-Hernández, J., Manzano-Méndez, F., Rodríguez-Ortiz, G., Romero Manzanares, A., \& Fuentes-López, M. (2014). Ecuaciones de biomasa aérea para Quercus laurina y Q. crassifolia en Oaxaca. Madera y Bosques, 20(2), 33-48. doi: 10.21829/myb.2014.202162

Ruppert, Ch., \& Antinori, A. (2008). Mexican and German Communal Forestry: An Accountability Framework for Comparing Governance. Governing Shared Resources: Connecting Local Experience to Global Challenges, 12th Biennial Conference of the International Association for the Study of the Commons. Cheltenham, Inglaterra.

Rzedowski, J. (1978). Vegetación de México. Ciudad de México, México: Limusa.

Rzedowski, J. (1991). Diversidad y orígenes de la flora fanerogámica de México. Acta Botánica Mexicana, 14, 3-21.

Seidl, R., Rammer, W., \& Spies, T. A. (2014). Disturbance legacies increase the resilience of forest ecosystem structure, composition, and functioning. Ecological Applications, 24, 2063-2077. doi: 10.1890/14-0255.1

Secretaría de Medio Ambiente y Recursos Naturales [Semarnat]. (2011). Anuario estadístico de la producción forestal 2008. México: Semarnat.

Secretaría de Medio Ambiente y Recursos Naturales [Semarnat]. (2014). Anuario estadístico de la producción forestal 2014. México: Semarnat.

Segura-Warnholtz, G. (2014). Quince años de políticas públicas para la acción colectiva en comunidades forestales. Revista Mexicana de Sociologia, 76(5), 105-135.

Silva-Arredondo, F., \& Návar-Cháidez, J. (2010). Factores de expansión de biomasa en comunidades forestales templadas del norte de Durango, México. Revista Mexicana de Ciencias Forestales, 1(1), 5562.

Summers, J. K., Smith, L. M., Case, J. L., \& Linthurst, R. A. (2012). A review of the elements of human well-being with an emphasis on the contribution of ecosystem services. Ambio, 41, 327-340. doi: 10.1007/s13280-012-0256-7.

Taylor, P. L., Cronkleton, P., \& Barry, D. (2011). Learning in the field: Using community self studies to strengthen forest based social movement. Sustainable Development, 21, 209-223. doi: $10.1002 /$ sd.498

TEEB (ed.). (2010). The economics of nature: a synthesis of the approach, conclusions and recommendations of TEEB, The economics of ecosystems \& biodiversity. Geneva: UNEP.

Torres, J. M., \& Guevara, A. (2002). El potencial de México para la producción de servicios ambientales: captura de carbono y desempeño hidráulico. Gaceta Ecológica, 63, 40-75 
Valencia, S. (2004). Diversidad del género Quercus (Fagaceae) en México. Boletín de la Sociedad Botánica de México, 75, 33-53.

Vázquez, V. (2015). Manejo forestal comunitario, gobernanza y género en Hidalgo, México. Revista mexicana de sociología, 77(4), 611-635.

Vela, G., López, J., \& Rodríguez, M. (2012). Niveles de carbono orgánico total en el Suelo de Conservación del Distrito Federal, centro de México. Investigaciones Geográficas, 77: 18-30.

Vesterdal, L., Clarke, N., Sigurdsson, B., \& Gunsersen, P. (2013). Do tree species influence soil carbon stocks in temperate and boreal forests? Forest Ecology and Management, 309, 4-18. doi: 10.1016/j.foreco.2013.01.017

Vihervaara, P., Rönkä, M., \& Walls, M. (2010). Trends in Ecosystem Service Research: Early Steps and Current Drivers. Ambio, 39, 314-324. doi: 10.1007/s13280-010-0048-x

Wallace, J., Aquilué, N., Archambault, Ch., Carpentier, S., Francoeur, X., Greffard, M. E., Laforest, L., Galicia, L., \& Messier, Ch. (2015). Present forest management structures and policies in temperate forests of Mexico: Challenges and prospects for unique tree species assemblages. Forest Chronicle, 91(3), 306-317. doi: $10.5558 /$ tfc $2015-052$

West, P., Igoe, J., \& Brockington, D. (2006). Parks and peoples: the social impact of protected areas. Annual Review of Anthropology, 35, 251277. doi:10.1146/annurev.anthro.35.081705.123308

Williams Linera, G. (1996). Crecimiento dimétrico de árboles caducifolios y perennifolios del bosque mesófilo de montaña en los alrededores de Xalapa. Madera y Bosques, (2) 2: 53-65.
Xiaogai, G., Lixiong, Z., Wenfa, X., Zhilin, H., Xiansheng, G., \& Benwang, G. (2013). Effect of litter substrate quality and soil nutrients on forest litter decomposition: A review. Acta Ecologica Sinica, 33, 102-108. doi: 10.1016/j.chnaes.2013.01.006

Zerecero, G., \& Pérez, V. M. (1981). El manejo del bosque y la industria forestal en el norte del país. Ciencia Forestal, 34(6), 30-42.

Manuscrito recibido el: 31 de octubre de 2016

Aceptado el: 28 de octubre de 2017

Publicado el: 12 de junio de 2018

Este documento se debe citar como:

Galicia, L., Chávez-Vergara, B., Kolb, M., Jasso-Flores, R. I., RodríguezBustos, L. A., Solís, E. L., Guerra de la Cruz, V., Pérez-Campuzano, E., \& Villanueva, A. (2018). Perspectivas del enfoque socioecológico en la conservación, el aprovechamiento y pago de servicios ambientales de los bosques templados de México. Madera y Bosques, 24(2), e2421443. doi: 10.21829/myb.2018.2421443

Madera y Bosques por Instituto de Ecología, A.C. se distribuye bajo una Licencia Creative Commons Atribución-NoComercialCompartirlgual 4.0 Internacional. 